\title{
Efectividad de una intervención en educación alimentaria y actividad física para prevenir obesidad en escolares de la ciudad de Casablanca, Chile (2003-2004)
}

\author{
Juliana Kain B1a, Ricardo U auy D 1,2, Bárbara Leyton D 1b, \\ Ricardo Cerda $\mathbf{R}^{3 \mathrm{C}}$, Sonia 0 livares $C^{1 \mathrm{C}}$, Fernando Vio $\mathrm{D}^{1}$. \\ Effectiveness of a dietary and physical \\ activity intervention to prevent \\ obesity in school age children
}

Background: With the aim of contributing to he Healthy Goal 2010 of reducing significantly the prevalence of childhood obestiy, we developed and implemented during 2003 and 2004, a school-based obesity prevention intervention which included nutrition education and the promotion of physical activity. Aim: To report the results of the intervention. Material and methods: The sample included 1760 children ( $1^{\text {st }}$ to $7^{\text {th }}$ grade) from 3 elementary public schools in Casablanca (experimental group) and 671 from a similar school located in Quillota, a neighboring city (control). Primary outcomes were body mass index (BMI) Z score, the mile and shuttle-run tests and obesity prevalence. We also compared changes in waist circumference and triceps skinfold between both groups. Effectiveness of the intervention was assessed by analyzing separately the group *age* time interaction for the first 3 outcomes (follow-up-baseline), using a mixed model of covariance and by comparing variations in obesity prevalence between both groups. Results: There was a significant decline in BMI Z scores in experimental schools for both genders, but greater in boys ( $p<0.001$ versus $p=0.0034$ in girls), while in controls, BMI Z scores increased. Obesity prevalence declined significantly in experimental schools; from 17 to $12.3 \%$ and from 14.1 to $10.3 \%$ in boys and girls respectively, while in the control group, it remained unchanged. Also, triceps skinfold in girls from Casablanca increased significantly less than that of control girls. Conclusion: This intervention proved that it is possible to reduce significantly the prevalence of obesity in Chilean schoolchildren attending public elementary schools (Rev Méd Chile 2008, 136: 22-30).

(Key words: Body mass index; Child, preschool; Obesity, Children, prevention)

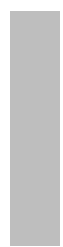

Recibido el 9 de marzo, 2007. Aceptado el 29 de agosto, 2007.

${ }^{1}$ Instituto de Nutrición y Tecnología de los Alimentos (INTA), Universidad de Chile. ${ }^{2}$ London School of Hygiene and Tropical Science. Londres, Inglaterra. ${ }^{3}$ Escuela de Nutrición, Facultad de Medicina, Universidad de Chile

aBioquímico, Master en Salud Pública

${ }^{b}$ Estadística

${ }^{\mathrm{c}}$ Nutricionista, Magíster en Nutrición Pública

Correspondencia a: Juliana Kain. INTA Universidad de Chile. Av. Macul 5540, Macul, Santiago. Fono: 97814953 Fax:

2214030. E mail: jkain@inta.cl 
$\mathrm{L}$ a prevalencia de obesidad infantil en Chile se ha triplicado en las dos últimas décadas; en escolares de primer año básico, ésta se incrementó desde 7\% en 1987 a 18,5\% en 2005 . El Consejo de Promoción de Salud (Vida Chile) ha establecido la reducción de la prevalencia en este grupo a $12 \%$ para el año $2010^{2}$. Como una forma de enfrentar el problema de la obesidad infantil, el Instituto de Nutrición y Tecnología de los Alimentos de la Universidad de Chile (INTA) en colaboración con Chiledeportes, los Ministerios de Educación y Salud y una empresa privada, diseñaron e implementaron en el año 2002 una intervención de prevención de obesidad en escolares de educación básica en las ciudades de Curicó, Santiago y Casablanca Los resultados de esta intervención mostraron una disminución significativa de la obesidad en los hombres, mientras que en las niñas, aun cuando se observó una reducción, el cambio no fue significativo respecto a lo ocurrido en las escuelas control ${ }^{3}$. Durante los años 2003 y 2004, se continuó con esta intervención sólo en los escolares de las tres escuelas de Casablanca. En esta oportunidad se muestra la efectividad de esta intervención basada en educación alimentaria y varias iniciativas en actividad física, en términos de cambios antropométricos y de aptitud física.

\section{Metodología}

Diseño. Estudio controlado no aleatorio en el cual se intervinieron durante 2 años los estudiantes de tres escuelas básicas municipalizadas de la ciudad de Casablanca y cuyos resultados fueron comparados con los obtenidos en escolares no intervenidos de una escuela de Quillota (misma región). Aun cuando Quillota es una ciudad con mayor número de habitantes que Casablanca (76.000 versus 16.000) y la proporción de población rural es menor $(13 \% \text { versus } 30 \%)^{4}$, los alumnos eran similares desde el punto de vista socioeconómico, determinado por el índice que utiliza la Junta Nacional de Auxiliar Escolar y Becas para determinar la vulnerabilidad escolar (IVE). Es así como el promedio de los IVE de las tres escuelas de Casablanca es prácticamente igual al de la escuela de Quillota (27,7 y 29 respectivamente en el $2004)^{5}$. Aun cuando nuestra intención fue efectuar un estudio aleatorio, esto no fue posible, ya que las autoridades de Casablanca nos indicaron que debíamos intervenir todas las escuelas básicas municipalizadas.

Sujetos. Participaron en este estudio todos los escolares (1ำ a 7o en 2003) de las escuelas básicas municipalizadas de Casablanca; estas escuelas son: Manuel Bravo, Domingo Ortiz y Arturo Echazarreta. La escuela Arauco de la ciudad de Quillota sirvió como control. Se incluyeron escolares entre $1^{\underline{0}}$ y $7^{\circ} \mathrm{o}$ básico en el seguimiento de dos años, ya que los de $8^{\circ}$ básico hubieran abandonado la escuela dentro del período del estudio. El número total de escolares intervenidos al inicio fue de 1.760 , mientras que los controles fueron 671. La muestra final incluida en este análisis fue de 1.466 escolares intervenidos y 573 escolares controles. Esta reducción $(16,7 \%$ en los intervenidos y $14,6 \%$ en los controles) se debió básicamente a la pérdida de niños por cambio de colegio y a aquéllos que no contaban con las mediciones completas en los dos años de seguimiento ( 4 mediciones). Como el número de sujetos intervenidos estaba definido a priori, se calculó el número de sujetos controles, asumiendo una diferencia en el IMC promedio de 0,7 unidades entre el grupo control y el intervenido al final de la intervención (aumento normal del IMC), con un alfa de 5\% y un poder de 80\%. El n resultante para el grupo control fue de 464 sujetos, es decir, el n del grupo control de nuestro estudio fue adecuado. Se presentan en esta oportunidad los resultados en base a las comparaciones de los valores finales con los iniciales.

Programa de intervención (se aplicó durante dos años escolares, equivalentes a 11 meses). A continuación se describen los componentes de la estrategia durante los años 2003 y 2004.

\section{Año 2003}

Alimentación y nutrición

- Los profesores (previamente capacitados por una nutricionista) aplicaron el programa educativo desarrollado por INTA/FAO ${ }^{6}$, cuyo objetivo es incorporar la educación en nutrición en el currículo de $3^{\circ}$ a $8^{\circ}$ años de la enseñanza básica.

- Charlas a los apoderados: la nutricionista efectuó dos charlas anuales con cada uno de los cursos entre $4^{\circ}$ y $8^{\circ}$ básicos, de las 3 escuelas. 
- Eventos comunitarios masivos, donde estuvo presente el tema alimentario, como por ejemplo, preparación de comidas saludables, diarios murales y construcción de pirámides alimentarias.

\section{Actividad Física}

- Incremento en 90 min semanales las clases de educación física para los alumnos entre $3^{\circ}$ y $8^{\circ}$ básicos. El tiempo extra se entregó a través de talleres deportivos.

- Aplicación del Programa Canadiense llamado Canadian Physical Activity Challenge (CALC) ${ }^{7}$ que fue adaptado a la realidad chilena por el MINSAL y MINEDUC y que consiste en motivar a los alumnos a incorporar la actividad física a su quehacer habitual. Fue aplicado por una profesora de educación física en los escolares entre $3^{\circ}$ y $6^{\circ}$ básico.

- Realización de actividades masivas con la familia, específicamente, cicletadas, caminatas a los cerros, campeonatos de ping-pong y otras similares.

- Capacitación para profesores de 1o a 4o básicos. Esta fue realizada por docentes de la Universidad de Playa Ancha de Valparaíso y consistió en cuatro sesiones teóricas para dar a conocer los distintos aspectos que debieran desarrollar los niños en las clases de educación física.

\section{Año 2004}

Durante el año 2004 se debieron realizar algunos cambios en la estrategia por falta de recursos; las actividades fueron las siguientes:

\section{Alimentación y nutrición}

- Se les solicitó a los profesores de $3^{\circ}$ a $7^{\circ}$ que realicen al menos cuatro actividades de las propuestas, por curso.

- Se le solicitó a la nutricionista del consultorio que efectuara charlas a los padres. Como la disponibilidad de esa profesional era limitada, se decidió, junto a los directores de las escuelas que estas charlas se las dirigieran a los padres de los niños obesos. El número de charlas varió entre una y tres por escuela.

\section{Actividad física}

- Se continuó con los 90 min adicionales semanales de clases de educación física.

- Se efectuó capacitación en terreno para mejorar la calidad de las clases de educación física de $1^{\mathrm{o}}$ y $2^{\mathrm{o}}$ básicos. Para ello se contrató una profesora de educación física que trabajó junto a nueve profesores de $1^{\circ}$ y $2^{2}$ básicos de las tres escuelas (entre 5 y 10 sesiones). El objetivo fue enseñarle a los profesores a organizar las clases de educación física en forma práctica, de tal forma de que los niños estuviesen una mayor cantidad de tiempo en movimiento.

Variables. Las principales variables de resultados (outcomes) en relación a estado nutricional son el puntaje Z de IMC y la prevalencia de obesidad, mientras que las relacionadas con aptitud física son las pruebas de la milla y Navette. Las variables secundarias corresponden a la circunferencia de cintura y al pliegue tricipital. Todas las mediciones se efectuaron en marzo y noviembre de 2003 y los mismos meses de 2004.

Mediciones antropométricas. Se efectuaron mediciones antropométricas de peso, talla, circunferencia de cintura y pliegue tricipital en todos los escolares. Estas mediciones fueron realizadas por una nutricionista entrenada (distinta a la que implementó el componente educativo).

Cuando se efectuaron las mediciones de peso y la talla a los alumnos se les solicitó que se quitaran zapatos, cinturones, jumpers o pantalones. Se utilizó una balanza SECA 840 con tallímetro incorporado (precisión $100 \mathrm{~g}$ y $0,1 \mathrm{~cm}$, respectivamente). Con estas mediciones, se calculó el IMC $\left(\mathrm{kg} / \mathrm{m}^{2}\right)$ de cada niño. Se clasificó a los niños como obesos si su IMC era $\geq p 95$ de la referencia CDC/NCHS, tal como lo recomienda el Ministerio de Salud para la determinación de obesidad en mayores de 6 años ${ }^{8}$. La circunferencia de cintura se midió con una huincha no elástica en el punto medio entre la costilla inferior y la cresta ilíaca, mientras que el pliegue tricipital se midió tres veces consecutivas con un caliper Lange de precisión $1 \mathrm{~mm}$, utilizándose el promedio de estas determinaciones.

Mediciones de aptitud física. Se aplicaron pruebas de resistencia aeróbica recomendadas según edad. Estos fueron: la prueba de la milla desde $1^{\text {o }}$ a $4^{\underline{0}}$ básicos ( $1^{\mathrm{o}}$ y $2^{\mathrm{o}}$ básicos los niños corrieron $1 / 4$ de milla y los de $3^{\circ}$ y $4^{\circ}$ básicos, $1 / 2$ milla) y la de Navette, indicada para mayores de 10 años ${ }^{9}$. La 
prueba de la milla consiste en medir el tiempo que se demora un niño corriendo, en alcanzar la distancia (en este caso 400 u 800 m). La utilización de esta prueba está recomendado por la Asociación Canadiense de Salud (Health Canada) ${ }^{10}$ y por el Programa Norteamericano llamado The President's Challenge ${ }^{11}$. La prueba de Navette es ampliamente utilizada tanto en Chile ${ }^{12,13}$ como en el extranjero ${ }^{14}$ y consiste en registrar el número de vueltas (períodos) que hace el niño corriendo $20 \mathrm{~m}$ ida y vuelta a una velocidad creciente ${ }^{15}$. Estas pruebas fueron aplicadas por dos profesores de educación física entrenados para ese efecto.

Estadística. Se calcularon los valores promedios y desviación estándar (DE) de las variables antropométricas y de aptitud física al inicio y final, según sexo. Se compararon estas variables entre los niños intervenidos y los controles, utilizando ANOVA y se comparó la prevalencia de obesidad entre los dos grupos, utilizando el test de proporciones. Se presentan aquí sólo las cifras iniciales y finales de las variables para mayor claridad, aunque en el análisis estadístico se incluyeron los cuatro tiempos de evaluación. En la determinación de la efectividad de la intervención en relación a obesidad, se consideró el puntaje $Z$ de IMC y la prevalencia de obesidad y en aptitud física, la prueba de la milla en los menores de 10 años y la de Navette para los mayores de10 años.
Para determinar el efecto de la intervención en relación al puntaje $Z$ de IMC, se consideró la interacción de las tres variables independientes: grupo *edad* tiempo de medición, controlando por el valor inicial, utilizando el modelo mixto de covarianza (Proc Mixed - SAS versión 8.2). Se consideró significativo si el p de la interacción triple era $<0,05$. En caso de que la interacción triple no fuera significativa, se verificó si la interacción grupo *tiempo* lo era; si ese resultado era positivo, se concluye que la edad afecta de igual forma al grupo intervenido como al control.

La comparación entre la prevalencia de obesidad al final de la intervención con la inicial se efectuó usando el test de proporciones.

Para las variables milla y Navette, sólo se consideró el resultado de la interacción doble grupo*tiempo, ya que las pruebas fueron aplicadas a grupos específicos de edad.

También se evaluaron los resultados de la intervención en términos de variables antropométricas adicionales (circunferencia de cintura pliegue tricipital) usando el procedimiento Proc Mixed de la misma manera descrita anteriormente.

\section{Resultados}

Comparación de los sujetos al inicio. Las Tablas 1 y 2 muestran las características antropométricas de los varones y niñas, respectivamente, participantes del

\section{Tabla 1. Características antropométricas en escolares hombres intervenidos y controles al inicio y fin de la intervención (promedio y (DE))}

\begin{tabular}{|c|c|c|c|c|c|}
\hline & \multicolumn{2}{|c|}{$\begin{array}{l}\text { Grupo intervenido } \\
\mathrm{N}=750\end{array}$} & \multicolumn{3}{|c|}{$\begin{array}{l}\text { Grupo control } \\
\mathrm{N}=348\end{array}$} \\
\hline & Inicio & Fin & Inicio & Fin & $\mathrm{p}$ \\
\hline \multirow[t]{2}{*}{ Edad (años) } & 10,0 & 11,6 & 9,9 & 11,5 & \\
\hline & $(2,3)$ & $(2,2)$ & $(2,1)$ & $(2,1)$ & \\
\hline \multirow[t]{2}{*}{ IMC } & 19 & 19,7 & 19,4 & 20,6 & \\
\hline & $(3,3)$ & $(3,2)$ & $(3,8)$ & $(3,7)$ & \\
\hline \multirow[t]{2}{*}{$\mathrm{Z}$ de IMC } & 0,62 & 0,53 & 0,67 & 0,72 & $\mathrm{p}<0,001^{\mathrm{a}}$ \\
\hline & (1) & $(0,95)$ & (1) & (1) & \\
\hline \multirow[t]{2}{*}{ Circ cintura $(\mathrm{cm})$} & 64,9 & 68 & 65,6 & 68,5 & NS \\
\hline & $(9,7)$ & $(8,8)$ & $(10,5)$ & $(9,4)$ & \\
\hline \multirow[t]{2}{*}{ Pliegue tricipital (mm) } & 12,2 & 14,17 & 13 & 15,6 & \\
\hline & $(5,8)$ & $(6,3$ & $(6,3)$ & $(6,8)$ & NS \\
\hline
\end{tabular}

a =interacción grupo*tiempo*edad. 
Tabla 2. C aracterísticas antropométricas en escolares mujeres intervenidas y controles al inicio y fin de la intervención (promedio y (DE))

\begin{tabular}{|c|c|c|c|c|c|}
\hline & \multicolumn{2}{|c|}{$\begin{array}{l}\text { Grupo intervenido } \\
\mathrm{N}=717\end{array}$} & \multicolumn{3}{|c|}{$\begin{array}{l}\text { Grupo control } \\
\mathrm{N}=225\end{array}$} \\
\hline & Inicio & Fin & Inicio & Fin & $\mathrm{p}$ \\
\hline Edad (años) & $\begin{array}{l}9,8 \\
(2,3)\end{array}$ & $\begin{array}{l}11,4 \\
(2,3)\end{array}$ & $\begin{array}{l}9,9 \\
(2,2)\end{array}$ & $\begin{array}{l}11,5 \\
(2,2)\end{array}$ & \\
\hline IMC & $\begin{array}{l}19,3 \\
(3,5)\end{array}$ & $\begin{array}{l}20,1 \\
(3,5)\end{array}$ & $\begin{array}{l}19,4 \\
(4)\end{array}$ & $\begin{array}{l}20,8 \\
(3,8)\end{array}$ & \\
\hline$Z$ de IMC & $\begin{array}{l}0,64 \\
(0,95)\end{array}$ & $\begin{array}{l}0,58 \\
(0,9)\end{array}$ & $\begin{array}{l}0,64 \\
\text { (1) }\end{array}$ & $\begin{array}{c}0,72 \\
(0,9)\end{array}$ & $\begin{array}{l}p=0,156^{a} \\
p=0,0034^{b}\end{array}$ \\
\hline Circ cintura $(\mathrm{cm})$ & $\begin{array}{l}64,9 \\
(9,9)\end{array}$ & $\begin{array}{l}67,7 \\
(9,1)\end{array}$ & $\begin{array}{l}64 \\
(10,2)\end{array}$ & $\begin{array}{l}67,3 \\
(9,1)\end{array}$ & NS \\
\hline Pliegue tricipital (mm) & $\begin{array}{l}14,8 \\
(5,9)\end{array}$ & $\begin{array}{l}17,5 \\
(6,1)\end{array}$ & $\begin{array}{l}14,7 \\
(5,7)\end{array}$ & $\begin{array}{l}18,9 \\
(6,2)\end{array}$ & $\mathrm{p}<0,0001^{\mathrm{a}}$ \\
\hline
\end{tabular}

a =interacción grupo*tiempo*edad. b =interacción grupo*tiempo.

estudio. El número de hombres intervenidos fue de 750 , mientras que los controles sumaron 348. En las niñas (Tabla 2), estas cifras fueron 717 y 225, respectivamente. La proporción total de niños según sexo mostró que ésta fue diferente; en el grupo intervenido, el porcentaje de hombres fue de 51,1, mientras que en el grupo control, éste fue de 60,7.

Comparación de las variables antropométricas y de aptitud física del grupo intervenido y control al inicio. La comparación de las variables antropométricas iniciales entre los dos grupos tanto en hombres y en mujeres, mostró que éstas eran similares (Tablas 1 y 2 ).
Respecto a los valores promedio iniciales de las pruebas de la milla y Navette en hombres como en mujeres (Tablas 3 y 4), la prueba de ANOVA tampoco mostró diferencias significativas entre el grupo intervenido y de control.

El porcentaje de obesidad inicial fue mayor en los hombres del grupo control (21,6\% versus $17 \%)$, sin embargo esta diferencia no fue significativa ( $\mathrm{p}=0,081$ ). En las niñas en cambio, la prevalencia de obesidad al inicio fue prácticamente igual en ambos grupos (14\%).

Efectividad de la intervención. Las Tablas 1 y 2 muestran las características antropométricas al

Tabla 3. Aptitud física en escolares hombres intervenidos y controles al inicio y final de la intervención, (promedio y (DE))

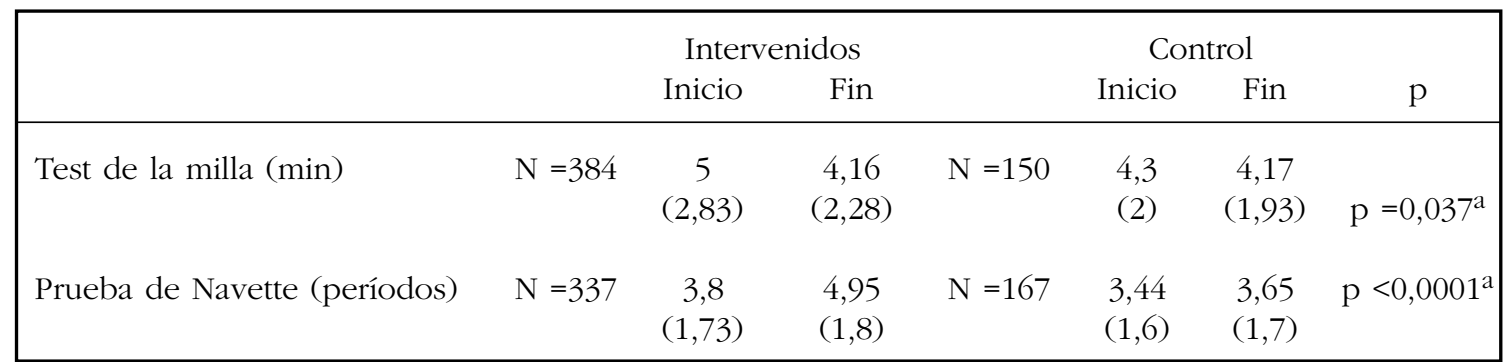

a =interacción grupo*tiempo. 
Tabla 4. Aptitud física en escolares mujeres intervenidas y controles al inicio y fin de la intervención (promedio y (DE))

\begin{tabular}{|lccccccc|}
\hline & & \multicolumn{4}{c|}{ Intervenidos } & \multicolumn{4}{c|}{ Control } \\
& & Inicio & Fin & & Inicio & Fin & $\mathrm{p}$ \\
\hline \multirow{2}{*}{ Test de la milla (min) } & $\mathrm{N}=377$ & 4,81 & 4,2 & $\mathrm{~N}=93$ & 4,43 & 4,2 & $\mathrm{p}=0,005^{\mathrm{a}}$ \\
& & $(2,57)$ & $(2,33)$ & & $(2,27)$ & $(2,35)$ & \\
Prueba de Navette (períodos) & $\mathrm{N}=307$ & 2,75 & 3,05 & $\mathrm{~N}=122$ & 2,65 & 2,49 & $\mathrm{p}=0,0007^{\mathrm{a}}$ \\
& & $(1,16)$ & $(1,2)$ & & $(1,06)$ & $(1,1)$ & \\
\hline
\end{tabular}

a =interacción grupo*tiempo.

inicio y al final de la intervención del grupo intervenido y del grupo control en hombres y mujeres, respectivamente. En hombres, se observó un descenso significativo del puntaje $Z$ de IMC ( 0,9 unidades) en el grupo intervenido, mientras que en el grupo control, éste aumentó en 0,5 unidades. Tanto la circunferencia de cintura como el pliegue tricipital aumentaron en ambos grupos; no hubo diferencia significativa en los incrementos de estas variables. En las niñas (Tabla 2), la disminución del puntaje $\mathrm{Z}$ de IMC del grupo intervenido fue menor que aquella observada en los varones, mientras que en el grupo control, el aumento fue similar. Se observó una diferencia significativa entre los dos grupos a través del tiempo, sin embargo, la interacción triple grupo "edad* tiempo no fue significativa. El pliegue tricipital en las niñas intervenidas aumentó significativamente menos que en las controles.

Las Tablas 3 y 4 muestran las cifras iniciales y finales de las pruebas de la milla y Navette. En los hombres (Tabla 3), el valor promedio de la prueba de la milla varió de 5 a 4,16 min, mientras que en el grupo control, no se observó variación. Es importante destacar que aunque no se encontró una diferencia significativa entre los valores promedio iniciales de los grupos intervenido y control, la cifra fue mayor en el grupo intervenido, es decir, la condición física era más deficiente en este grupo. Además, aunque el cambio experimentado fue significativamente mejor en el grupo intervenido, ambos grupos tuvieron el mismo valor final. En la prueba de Navette, la mejoría del grupo intervenido fue significativa (un valor mayor indica mejor condición física), ya que en promedio, los hombres fueron capaces de correr 1 período adicional, mientras que en el grupo control, el valor final fue similar. En las niñas (Tabla 4), tanto para la prueba de la milla como para Navette, la tendencia fue muy parecida a la observada en los hombres, sin embargo la mejoría del grupo intervenido fue mucho menor. También para la prueba de Navette, los valores fueron menores que en los varones, es decir la condición física en las niñas estaba más deteriorada.

Las Figuras 1 y 2 muestran la variación del porcentaje de obesidad entre la línea base y el término de la intervención. En los varones (Figura 1), este porcentaje disminuyó desde $17 \%$ a $12,3 \%$, mientras que en los controles, la prevalencia se mantuvo igual; en las niñas (Figura 2), la disminución en el grupo intervenido fue de 4 puntos porcentuales, mientras que en el grupo control, este porcentaje no varió.

\section{DisCUSIÓN}

El objetivo principal de este estudio fue evaluar en forma controlada los efectos de una intervención que incluyó educación en alimentación y nutrición y fomento de la actividad física. Para detectar efectividad (evaluación de impacto), seleccionamos variables relacionadas con estas dos áreas, específicamente el Z de IMC, la prevalencia de obesidad y las pruebas de milla y Navette.

El promedio del Z de IMC al inicio, tanto en varones como en las niñas, fue significativamente mayor respecto a la referencia $\mathrm{CDC}^{16}$; esta cifra está asociada a la alta prevalencia de obesidad en los escolares del país. La cifra inicial de circunferencia de cintura (asociada a distribución de grasa abdominal) de los hombres intervenidos es similar a la del promedio de varones de 10 años incluidos en la Encuesta NHANES III efectuada entre 1988 y $1994^{17}$, 


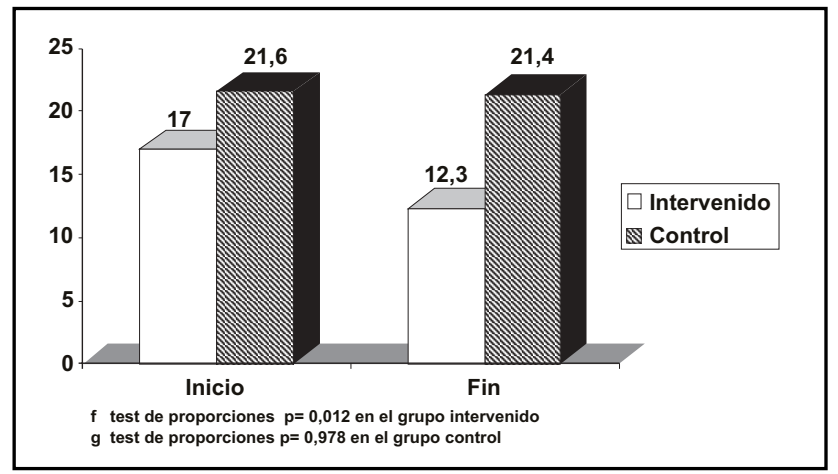

Figura 1. Prevalencia de obesidad al inicio y fin de la intervención en escolares hombres intervenidos y controles (\%).

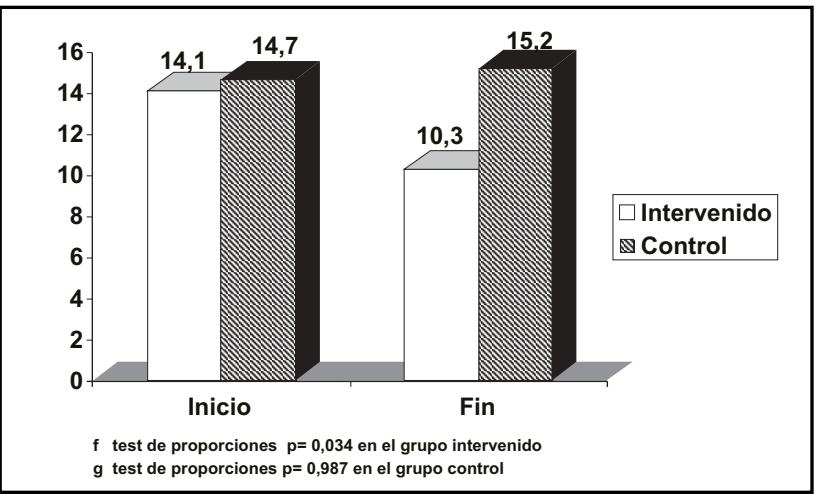

Figura 2. Prevalencia de obesidad al inicio y fin de la intervención en escolares mujeres intervenidas y controles (\%).

64,9 comparado con $65,1 \mathrm{~cm}$. En el caso de las niñas intervenidas, la cifra promedio inicial de este estudio, también es similar a la de las norteamericanas de la misma edad, 64,9 y $64,7 \mathrm{~cm}$, respectivamente. En relación al pliegue tricipital (asociado a grasa total), la misma comparación muestra valores similares a los niños norteamericanos (12,2 y $12,5 \mathrm{~mm}$ en los hombres y 14,8 y $15 \mathrm{~mm}$ en las niñas). Estos resultados muestran que los niños chilenos tienen similar porcentaje de grasa abdominal y corporal a niños norteamericanos de alrededor de 10 años.

Las pruebas de aptitud física aplicadas en niños incluyen medidas para evaluar resistencia aeróbica, fuerza muscular y flexibilidad. Por recomendación de expertos y por tiempo, seleccionamos sólo pruebas de resistencia aeróbica: la de la milla para los niños $<10$ años y Navette para los >10 años. Ambas han sido utilizadas ampliamente; la primera es parte de la batería de pruebas de aptitud física nacionales de Canadá y Estados Unidos de Norteamérica con valores referenciales según edad y sexo, mientras que la segunda se utiliza ampliamente en Europa (ya que está incluida en el Eurofit) y también en Chile. Al comparar las cifras iniciales de la prueba de la milla de este estudio según edad y sexo, con los de la referencia canadiense (percentil 50) se observó que en los niños de 6 y 7 años (ambos sexos), los resultados fueron muy similares a los de la referencia, mientras que en los de 8 y 9 años, las cifras de este estudio fueron significativamente peores. En la prueba de Navette, nuestros resultados fueron más deficientes que los de la referencia chilena (basadas en la distribución percentilar obtenida de su estudio de 10.600 sujetos efectuado en 1998), especialmente en las niñas ${ }^{13}$.

Los resultados de este estudio muestran que la intervención que se aplicó en los escolares de Casablanca fue muy efectiva. En los varones, mientras el Z de IMC disminuyó significativamente en el grupo intervenido, en el grupo control se observó lo contrario. En las niñas, los cambios siguieron la misma dirección, pero en el grupo intervenido el 
cambio fue menor, hecho que muestra el resultado de la interacción grupo* tiempo, cuyo p resultó ser más significativo en los varones. La efectividad medida por la prevalencia de obesidad, también muestra una baja notable en este parámetro en los dos años de intervención, mientras que en el grupo control, la proporción de obesos se mantuvo constante. En un seguimiento longitudinal de tres años (2002-2004) realizado sólo con los escolares de Casablanca, se demostró que la prevalencia de obesidad se redujo de $21,6 \%$ a $12,2 \%$ en los hombres y de $19,4 \%$ a $8,7 \%$ en las niñas ${ }^{18}$.

Los resultados de la prueba de Navette fueron igualmente buenos en nuestro estudio en las tres comunas en 2002, como en el que se presentan aquí, sin embargo, en el primero se logró en 6 meses, lo mismo que se obtuvo en el actual, después de 11 meses de intervención. Una de las posibles explicaciones podría ser que la falta de motivación por parte de los niños de efectuar la prueba tras realizarla en varias oportunidades anteriores. Se ha reportado que existen una serie de variables confundentes que pueden afectar los resultados de las pruebas que miden aptitud física. Estas incluyen variaciones en la motivación, condiciones externas, dinámica del grupo y valorización percibida de la prueba ${ }^{19}$. Nosotros detectamos mayor dificultad en lograr que los escolares efectuaran esta prueba como corresponde al final de la intervención.

La mejoría en la aptitud física fue mayor en los hombres; en las prueba de la milla y Navette, ellos mejoraron $17 \%$ y $30 \%$, respectivamente, mientras las niñas estas cifras fueron $12 \%$ y $11 \%$. Esto se podrá deber a que los hombres son más activos en las clases de educación física, lo que se traduce en mayor gasto energético. Varios estudios han mostrado que las niñas son menos activas que los varones y que es menor el tiempo que destinan a actividad moderada e intensa en las clases de educación física $^{20,21}$. Vale la pena recalcar sobre la deficiente condición física de los escolares chilenos que nosotros ya habíamos reportado previamente ${ }^{22}$.

En relación a las variables secundarias, hubo un cambio significativo en el pliegue tricipital en las niñas (menor incremento en las intervenidas) contrastando con el hecho de que el cambio en $Z$ de BMI fue mayor en los varones y el de la circunferencia de cintura fue similar en ambos grupos. Esto podría deberse a que las niñas tienden a acumular grasa durante la pubertad en forma diferente a los hombres, este fenómeno se establece en el período prepuberal $^{23}$. En los hombres en cambio, el IMC tiene más fuerza que las diferencias en la distribución de grasa corporal ${ }^{24}$. Durante el desarrollo de la intervención alrededor de $50 \%$ de los niños tuvieron su pubertad; el estadio de desarrollo según Tanner no fue determinado por ser ésta una intervención con un foco de salud pública y no de caso individual. El contar con un grupo comparativo de edad semejante equilibra este efecto en ambos grupos. La pubertad indudablemente se relaciona con la ganancia de peso magro y graso, y la distribución de grasa corporal.

Es necesario reconocer que en el estudio que se está presentando aquí los escolares de Casablanca ya habían sido intervenidos el año anterior (2002) y los resultados mostraron una disminución considerable en la prevalencia de obesidad ${ }^{18}$, sin embargo el análisis actual es controlado y tal como se observa en las Tablas 1 y 2 , no se encontraron diferencias significativas entre las variables primarias ni en las secundarias al inicio. Además el análisis estadístico Proc Mixed no sólo considera el cambio de las variables en el tiempo, sino que controla por el valor inicial. La asignación intencional de la intervención por las autoridades educacionales, sin permitir que este estudio tenga un diseño aleatorio, constituye una limitación y lo mejor que se pudo hacer fue contar con una escuela control con similar infraestructura y con niños de características socioeconómicas parecidas.

La importancia fundamental de los resultados obtenidos en este estudio, es la factibilidad de aplicar las intervenciones, tanto en el aspecto alimentario como de actividad física. En el caso de la intervención educativa en alimentación y nutrición, como la reforma educacional no ha incorporado nuevos programas al currículo de la enseñanza básica, la estrategia consideró los objetivos de los planes y programas de estudios y las teorías y modelos educativos recomendados internacionalmente para lograr cambios de conducta elaborada como complemento al currículo ${ }^{6}$. En el caso de la actividad física, se aumentaron las clases de educación física, una iniciativa absolutamente necesaria, ya que los escolares sólo tienen, por currículo, una sesión semanal de educación física consistente en dos clases de 45 min cada una. No recomendamos aplicar el CALC, ya que los profesores no se hicieron cargo del programa argumentan- 
do falta de tiempo, pero proponemos la capacitación de los profesores unidocentes para mejorar las clases de educación física, experiencia muy positiva para lograr aumentar el tiempo de movimiento de los niños.

\section{REFERENCIAS}

1. Junta Nacional de Auxilio Escolar y Becas de Chile (JUNAEB) (www.junaeb.cl)

2. Salinas J, Vio F. Promoción de la salud y actividad física en Chile. Rev PANAM Salud Pública 2003; 33: 64-9.

3. Kain J, Uauy R, albala C, Vio F, Cerda R, Leyton B. School-based obesity prevention in Chilean school children: methodology and evaluation of a controlled study. IJO 2004; 28: 483-93.

4. InstTtuto Nacional de Estadísticas de Chite: www.ine.cl. Consultado en junio 2007.

5. Minsterio de EduCaCión de ChIIE: www.mineduc.cl. Consultado en junio 2007.

6. Olivares S, Morón C, Kain J, Zacarias I, Andrade M, LERA L ET AL. Propuesta metodológica para incorporar la educación en nutrición en la enseñanza básica: la experiencia chilena. Arch Latinoam Nutr 2004; 54 (Supl 1): 33-9.

7. CALC Program. Pag Web: www.cahperd.ca/calc/ index.html. Consultado en agosto 2006.

8. Pizarro T, Rodríguez L, Benayides X, Atalah E, MardoNES F, Rozowski J ET AL. Norma técnica de evaluación nutricional del niño de 6 a 18 años. Año 2003. Rev Chil Nutr 2004; 31: 128-37.

9. Leger la, Mercier D, Gadoury C, Lambert J. The multistage 20 metre shuttle run test for aerobic fitness. J Sports Sci 1988; 6: 93-101.

10. Canada Fitness Award Program, Health Canada (www.phac.aspc.gc.ca). Consultado en mayo 2006.

11. The President's Challenge: physical activity and fitness awards program (www.fitness.gov). Consultado en mayo 2006.

12. Sociedad Médica Educacional Ltda. Estudio para la elaboración del Plan 2001-2006. Programa Formación para el Deporte Digeder. Informe Final, agosto 2000.

13. Sociedad Médica Educacional Ltda. Aplicación Batería de Pruebas de Aptitud Física a Población Escolar Nacional 2001. Informe Final presentado a Digeder. Santiago, 2002.

\section{Agradecimientos}

Los autores desean agradecer a la Empresa Tresmontes-Lucchetti, Chiledeportes, Ministerio de Educación (MINEDUC) y Unidad de Promoción de Salud del Ministerio de Salud por el apoyo brindado para llevar a cabo este estudio.

14. Manios Y, Kafatos A, Mamalakis G. The effects of a health education intervention initiated at first grade over a 3 year period: physical activity and fitness indices. Health Education Research 1998; 13: 593606.

15. Vanhees L, Lefevre J, Philippaerts R, Martens M, Huygens W, Troosters T, Beunen G. How to assess physical activity? How to assess physical fitness? Eur J Cardiovasc Prev Rehabil 2005; 12: 102-14.

16. CDC/NCHS CDC 2000 Growth Charts: United States. http://www.chc.gov/growthcharts. Consultado en marzo 2006.

17. National Health and Nutrition Examination Data, United States, 1988-1994 (www.cdc.gov). Consultado en agosto 2006.

18. Kain J, Vio F, Leyton B, Cerda R, Olinares S, Uauy R, Albala C. Estrategia de promoción de la salud en escolares de educación básica municipalizada de la comunidad de Casablanca, Chile. Rev Chil Nutr 2005; 32: $126-32$.

19. Naughton G, Carlson J, Greene D. Challenge to fitness testing in primary schools. J Sci Med Sport 2006; 9: 40-5.

20. Mc Kenzie T, Salus J, Faucette N, Roby J, Kolody B. Effects of a curriculum and inservice program on the quality of elementary physical education classes. Res Q Exer sport 1993; 64: 178-87.

21. Pate R, Freedson P, Sallis J, Taylor W, Sirand J, Frost S, DOWDA M. Compliance with physical activity guidelines: prevalence in population of children and youth. Ann Epidemiol 2002; 12: 303-8.

22. Kain J, Olivares S, Romo M, Leyton B, Vio F, Cerda R et AL. Estado nutricional y resistencia aeróbica en escolares de educación básica: línea base de un proyecto de Promoción de la Salud. Rev Méd Chile 2004; 132: 1395-1402.

23. Moreno L, Fieta J, Sarria A, Rodríguez G, Gil C, Bueno M. Secular changes in body fat patterning in children and adolescents of Zaragoza, Spain 1980-1995. ISO 2001; 25: 1656-1660.

24. Gultekin T, AkIN G, Ozer B. Gender differences in fat patterning in children living in Ankara. Anthropol Anz 2005; 63: 427-37. 